\title{
The Baikal neutrino experiment: Status, selected physics results, and perspectives
}

V. Aynutdinov ${ }^{\text {a }}$ A. Avrorin ${ }^{\mathrm{a}}$, V. Balkanov ${ }^{\mathrm{a}}$, I. Belolaptikov ${ }^{\mathrm{d}}$, N. Budnev ${ }^{\mathrm{b}}$, I. Danilchenko ${ }^{\mathrm{a}}$, G. Domogatsky ${ }^{\mathrm{a}}$, A. Doroshenko ${ }^{\mathrm{a}}$, A. Dyachok ${ }^{\mathrm{b}}$, Zh.-A. Dzhilkibaev $^{\mathrm{a}, *}$, S. Fialkovsky $^{\mathrm{f}}$, O. Gaponenko ${ }^{\mathrm{a}}$, K. Golubkov ${ }^{\mathrm{d}}$, O. Gress ${ }^{\mathrm{b}}$, T. Gress ${ }^{\mathrm{b}}$, O. Grishin ${ }^{\mathrm{b}}$, A. Klabukov ${ }^{\mathrm{a}}$, A. Klimov ${ }^{\mathrm{h}}$, A. Kochanov ${ }^{\text {, }}$, K. Konischev ${ }^{\text {d }}$, A. Koshechkin ${ }^{\mathrm{a}}$, V. Kulepov ${ }^{\mathrm{f}}$, L. Kuzmichev ${ }^{\mathrm{c}}$, E. Middell ${ }^{\mathrm{e}}$, S. Mikheyev ${ }^{\mathrm{a}}$, M. Milenin ${ }^{\mathrm{f}}$, R. Mirgazov ${ }^{\mathrm{b}}$, E. Osipova ${ }^{\mathrm{c}}$, G. Pan'kov ${ }^{\mathrm{b}}$, L. Pan'kov' ${ }^{\mathrm{b}}$, A. Panfilov ${ }^{\mathrm{a}}$, D. Petukhov ${ }^{\mathrm{a}}$, E. Pliskovsky ${ }^{\mathrm{d}}$, P. Pokhil ${ }^{\mathrm{a}}$, V. Poleschuk ${ }^{\mathrm{a}}$, E. Popova ${ }^{\mathrm{c}}$, V. Prosin ${ }^{\mathrm{c}}$, M. Rozanov ${ }^{\text {, V V Rubtzov }}{ }^{\mathrm{b}}$, A. Sheifler ${ }^{\mathrm{a}}$, A. Shirokov ${ }^{\mathrm{c}}$, B. Shoibonov ${ }^{\mathrm{d}}$, Ch. Spiering ${ }^{\mathrm{e}}$,

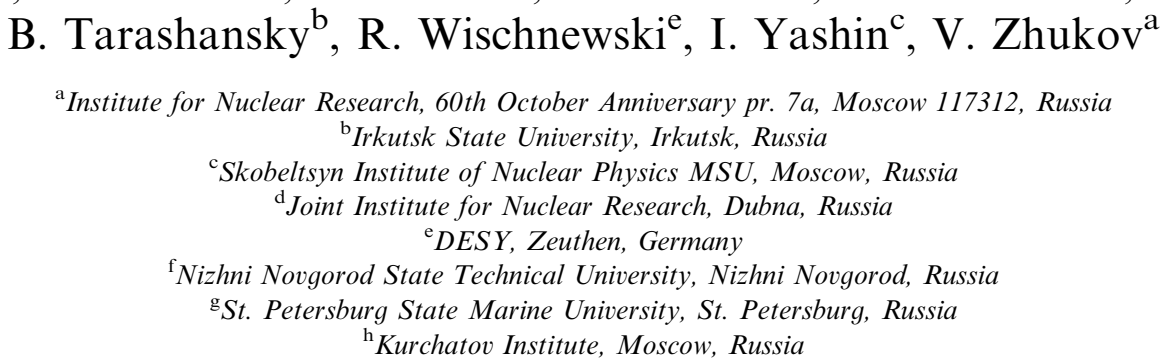

\begin{abstract}
We review the status of the Baikal neutrino telescope, which is operating in Lake Baikal since 1998 and has been upgraded to the 10 Mton detector NT200+ in 2005. We present selected physics results on searches for upward going neutrinos, relativistic magnetic monopoles and for very high-energy neutrinos. We describe the strategy of creating a detector on the Gigaton $\left(\mathrm{km}^{3}\right)$ scale at Lake Baikal. First steps of activities towards a $\mathrm{km}^{3}$ Baikal neutrino telescope are discussed.
\end{abstract}

(C) 2008 Elsevier B.V. All rights reserved.

PACS: 95.55.Vj; 95.85.Ry; 96.40.Tv

Keywords: Neutrino telescopes; Neutrino astronomy; UHE neutrinos; Baikal

\section{Introduction}

The Baikal neutrino telescope NT200 takes data since April 1998. On April 9th, 2005, the 10-Mton scale detector NT200+ was put into operation in Lake Baikal. Description of site properties, detector configuration and performance have been described elsewhere [1-5].

In this paper we review the current status of the Baikal neutrino experiment and the activities toward the

\footnotetext{
${ }^{*}$ Corresponding author.

E-mail address: djilkib@pcbai10.inr.ruhep.ru (Zh.-A. Dzhilkibaev).
}

$\mathrm{km}^{3}$-scale detector [6], as well as results obtained from the analysis of data taken with the Baikal neutrino telescope NT200 between April 1998 and February 2003 [7].

\section{Selected results obtained with NT200}

\subsection{Atmospheric neutrinos}

The signature of charged current muon neutrino events is a muon crossing the detector from below. Muon track reconstruction algorithms and background rejection have 
been described elsewhere [8]. Compared to Ref. [8] the analysis of the four-year sample (1038 days live time) was optimized for higher signal passing rate, and accepting a slightly higher contamination of $15-20 \%$ fake events [9]. A total of 372 upward going neutrino candidates were selected. From Monte-Carlo simulation a total of 385 atmospheric neutrino and background events are expected. The skyplot of these events is shown in Fig. 1.

\subsection{Search for neutrinos from WIMP annihilation}

The search for WIMPs with the Baikal neutrino telescope is based on a possible signal of nearly vertically upward going muons, exceeding the flux of atmospheric neutrinos. The method of event selection relies on the application of a series of cuts which are tailored to the response of the telescope to nearly vertically upward moving muons [10]. The applied cuts select muons with $-1<\cos (\theta)<-0.75$ and result in a detection area of about $1800 \mathrm{~m}^{2}$ for vertically upward going muons. The energy threshold for this analysis is $E_{\mathrm{thr}} \sim 10 \mathrm{GeV}$ i.e. significantly lower than for the analysis described in Section $2.1\left(E_{\mathrm{thr}} \sim 15-20 \mathrm{GeV}\right)$. Therefore the effect of oscillations is stronger visible. We expect a muon event suppression of $(25-30) \%$ due to neutrino oscillations assuming $\delta m^{2}=2.5 \times 10^{-3} \mathrm{eV}^{2}$ with full mixing, $\theta_{\mathrm{m}} \approx \pi / 4$.

From 1038 days of effective data taking between April 1998 and February 2003, 48 events with $-1<\cos (\theta)<$ -0.75 have been selected as clear neutrino events, compared to 56.6 events expected from atmospheric neutrinos in case of oscillations and 73.1 events without oscillations. The angular distribution of these events as well as the MC-predicted distributions are shown in Fig. 2. For the MC simulations we used the Bartol96 atmospheric neutrino flux [11] without (dashed curve) and with (solid curve) oscillations. Within statistical uncertainties the experimental angular distribution is consistent with the prediction including neutrino oscillations.

Regarding the 48 detected events as being induced by atmospheric neutrinos, one can derive an upper limit on the additional flux of muons from the center of the Earth due to annihilation of neutralinos - the favored candidate

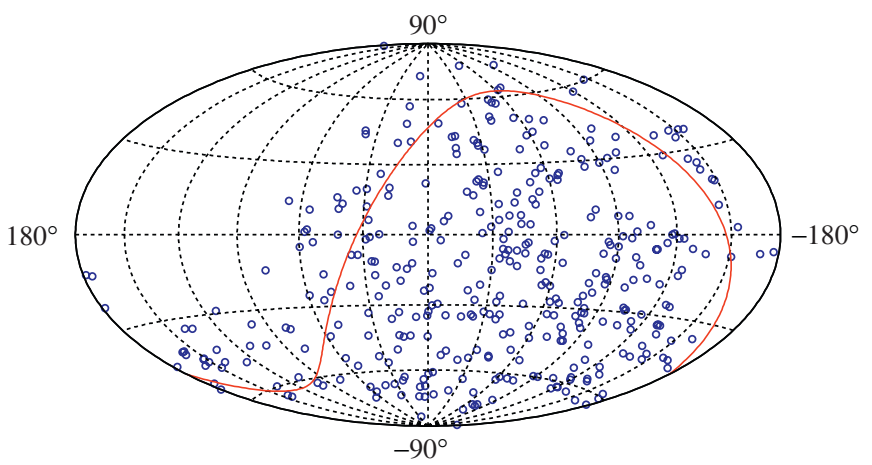

Fig. 1. Skyplot (galactic coordinates) of neutrino events for five years. The solid curve shows the equator.

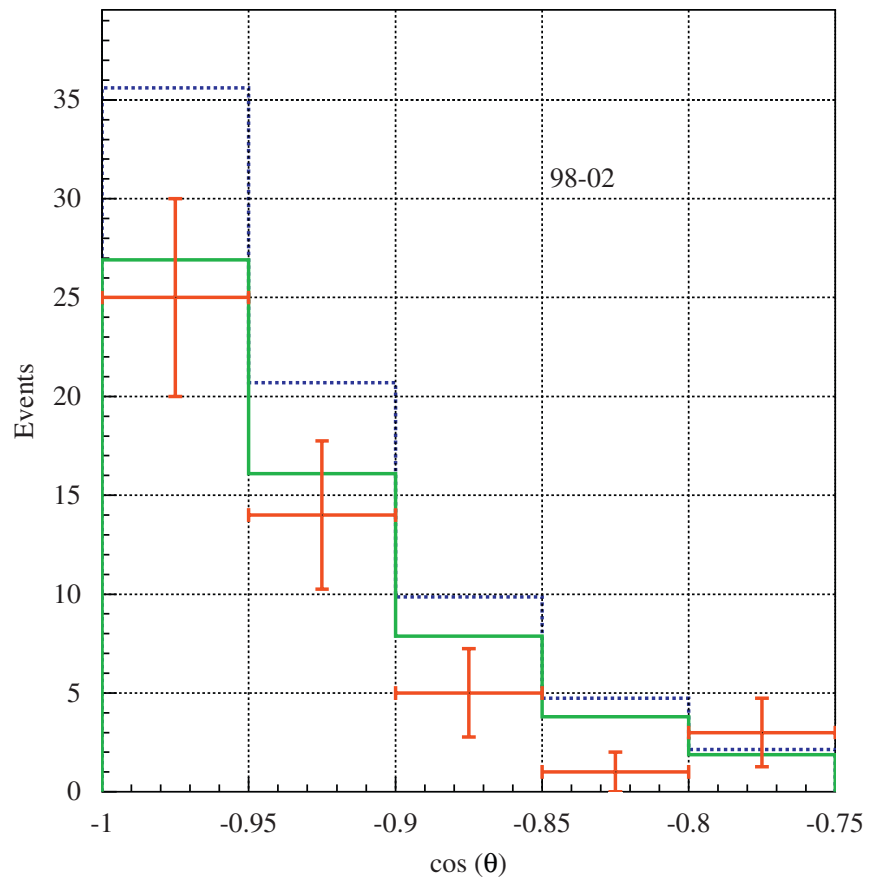

Fig. 2. Angular distributions of selected neutrino candidates as well as expected distributions in a case with and without oscillations (solid and dashed curves, respectively).

for cold dark matter. The $90 \%$ CL muon flux limits for six cones around the opposite zenith obtained with NT200 $\left(E_{\mathrm{thr}}>10 \mathrm{GeV}\right)$ in $1998-2002$ are shown in Fig. 3. It was shown [12-14] that the size of a cone which contains $90 \%$ of signal strongly depends on neutralino mass. The $90 \%$ CL flux limits are calculated as a function of neutralino mass using cones which collect $90 \%$ of the expected signal and are corrected for the $90 \%$ collection efficiency due to cone size. Also a correction is applied for each neutralino mass to translate from 10 to $1 \mathrm{GeV}$ threshold (thus modifying the results as presented earlier for $10 \mathrm{GeV}$ threshold [16]). These limits are shown in Fig. 4. Also shown in Figs. 3 and 4 are limits obtained by Baksan [12], MACRO [13], Super-Kamiokande [14] and AMANDA (from the hard neutralino annihilation channels) [15].

\subsection{A search for fast magnetic monopoles}

Fast magnetic monopoles with Dirac charge $g=68.5 e$ are interesting objects to search for with deep underwater neutrino telescopes. The intensity of monopole Cherenkov radiation is $\approx 8300$ times higher than that of muons. Optical modules of the Baikal experiment can detect such an object from a distance up to $100 \mathrm{~m}$. The processing chain for fast monopoles starts with the selection of events with a high multiplicity of hit channels: $N_{\text {hit }}>30$. In order to reduce the background from downward atmospheric muons we restrict ourself to monopoles coming from the lower hemisphere. For an upward going particle the times of hit channels increase with rising $z$-coordinates from bottom to top of the detector. To suppress downward 


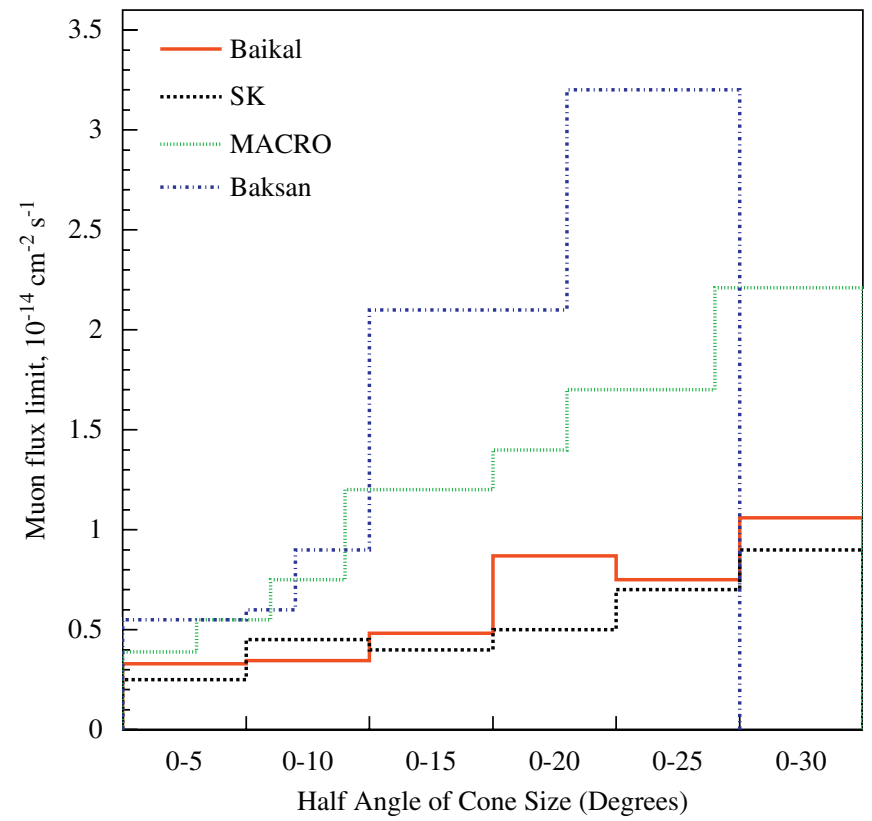

Fig. 3. Limits on the excess muon flux from the center of the Earth versus half-cone of the search angle.

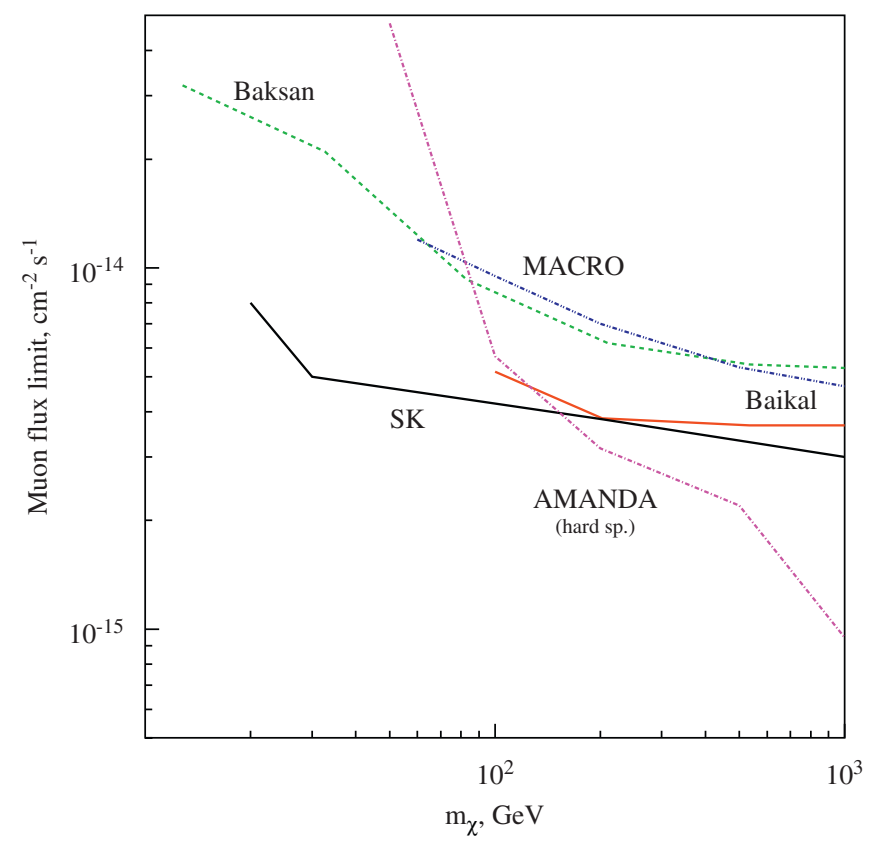

Fig. 4. Limits on the excess muon flux from the center of the Earth as a function of neutralino mass.

moving particles, a cut on the value of the time- $z$ correlation, $C_{t z}$, is applied:

$C_{t z}=\frac{\sum_{i=1}^{N_{\text {hit }}}\left(t_{i}-\bar{t}\right)\left(z_{i}-\bar{z}\right)}{N_{\text {hit }} \sigma_{t} \sigma_{z}}>0$

where $t_{i}$ and $z_{i}$ are time and $z$-coordinate of a fired channel, $\bar{t}$ and $\bar{z}$ are mean values for times and $z$-coordinates of the event and $\sigma_{t}$ and $\sigma_{z}$ the rms-errors for time and $z$-coordinates.

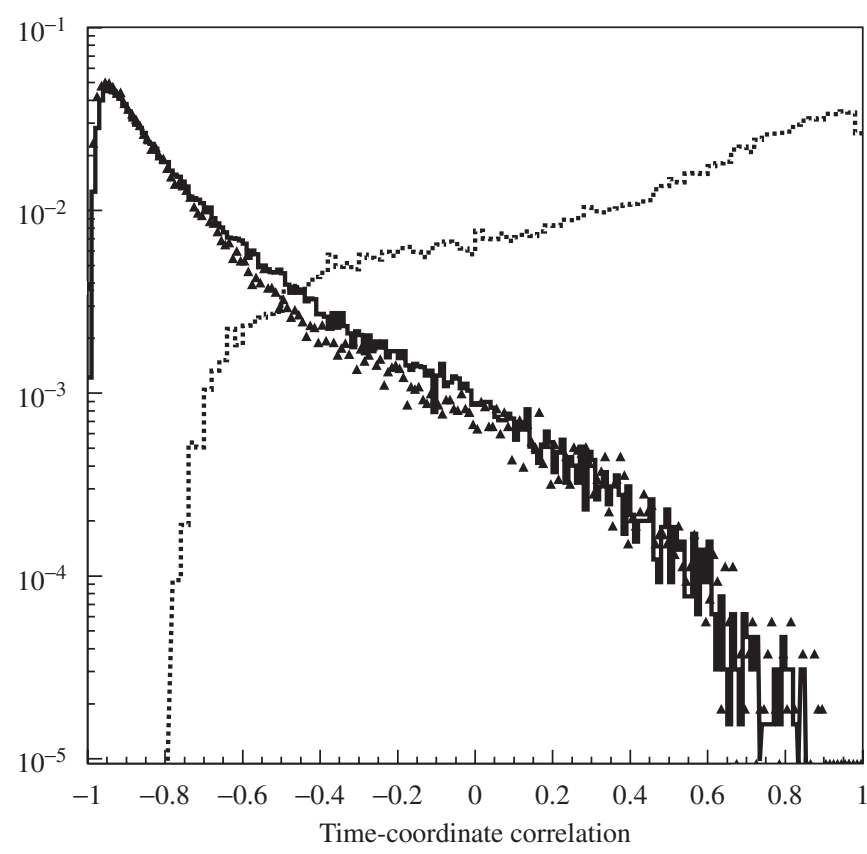

Fig. 5. $C_{t z}$ distributions for experimental events (triangles), simulated atmospheric muon events (solid), and simulated upward moving relativistic magnetic monopoles (dotted); multiplicity cut $N_{\text {hit }}>30$.

In Fig. 5 we compare the $C_{t z}$ distribution for experimental data (triangles) and simulated atmospheric muon events (solid curve) with simulated upward moving monopole events (dotted curve).

Within 994 days of live time using in this analysis, about $3 \times 10^{8}$ events with $N_{\text {hit }}>4$ have been recorded, with 20943 of them satisfying cut $0\left(N_{\text {hit }}>30\right.$ and $\left.C_{t z}>0\right)$. For further background suppression (see Ref. [17] for details of the analysis) we use additional cuts, which essentially reject muon events and at the same time only slightly reduce the effective area for relativistic monopoles ${ }^{1}$ :

(1) $N_{\text {hit }}>35$ and $C_{t z}>0.4-0.6$.

(2) $\chi^{2}$ determined from reconstruction has to be smaller than 3.

(3) Reconstructed zenith angle $\theta>100^{\circ}$.

(4) Reconstructed track distance from NT200 center $R_{\text {rec }}>20-25 \mathrm{~m}$.

No events from the experimental sample pass cuts (1)-(4). The acceptances $A_{\text {eff }}$ for monopoles with $\beta=1$, 0.9 and 0.8 have been calculated for all NT200 operation configurations (various sets of operating channels). For the time periods included, $A_{\text {eff }}$ varies between $3 \times 10^{8}$ and $6 \times$ $10^{8} \mathrm{~cm}^{2} \mathrm{sr}$ (for $\beta=1$ ). From the non-observation of candidate events in NT200 and the earlier stage telescopes NT36 and NT96 [18], a combined upper limit on the flux of fast monopoles with $90 \% \mathrm{CL}$ is obtained.

\footnotetext{
${ }^{1}$ Different values of cuts correspond to different NT200 operation configurations.
} 


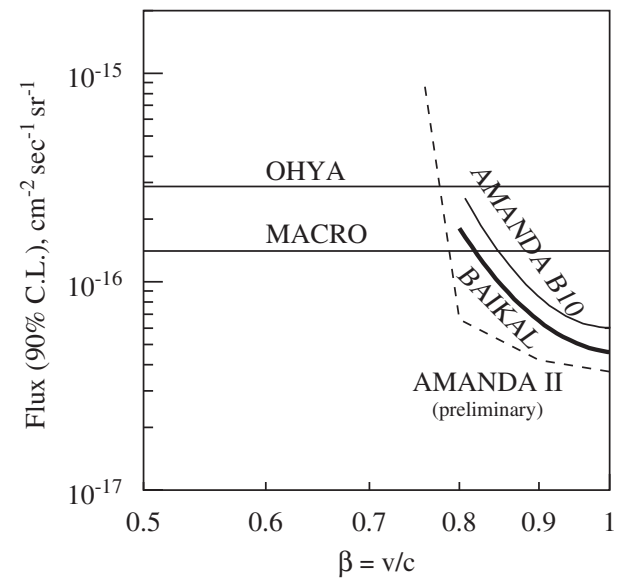

Fig. 6. Upper limits on the flux of fast monopoles obtained in this analysis (Baikal) and in other experiments.

Upper limit on a flux of magnetic monopoles with $\beta=1$ is $4.6 \times 10^{-17} \mathrm{~cm}^{-2} \mathrm{~s}^{-1} \mathrm{sr}^{-1}$. In Fig. 6 we compare our upper limit for an isotropic flux of fast monopoles obtained with the Baikal neutrino telescope to the limits from the underground experiments Ohya [19] and MACRO [20] and to the limit reported for the underice detector AMANDA B10 [21] and preliminary limit for AMANDA II [22].

\subsection{A search for extraterrestrial high-energy neutrinos}

The Baikal survey for high-energy neutrinos searches for bright cascades produced at the neutrino interaction vertex in a large volume around the neutrino telescope [3]. We select events with high multiplicity of hit channels $N_{\text {hit }}$, corresponding to bright cascades. To separate high-energy neutrino events from background events a cut to select events with upward moving light signals has been developed. We define for each event $t_{\min }=\min \left(t_{i}-t_{j}\right)$, where $t_{i}, t_{j}$ are the arrival times at channels $i, j$ on each string, and the minimum over all strings is calculated. Positive and negative values of $t_{\min }$ correspond to upward and downward propagation of light, respectively.

Within the 1038 days of the detector live time between April 1998 and February 2003, $3.45 \times 10^{8}$ events with $N_{\text {hit }} \geqslant 4$ have been recorded. For this analysis we used 22597 events with hit channel multiplicity $N_{\text {hit }}>15$ and $t_{\min }>-10 \mathrm{~ns}$. We conclude that data are consistent with simulated background for both $t_{\min }$ and $N_{\text {hit }}$ distributions. No statistically significant excess above the background from atmospheric muons has been observed. To maximize the sensitivity to a neutrino signal we introduce a cut in the $\left(t_{\min }, N_{\text {hit }}\right)$ phase space.

Since no events have been observed which pass the final cuts upper limits on the diffuse flux of extraterrestrial neutrinos are calculated. For a $90 \%$ confidence level an upper limit on the number of signal events of $n_{90 \%}=2.5$ is
Table 1

Expected number of events $N_{\mathrm{m}}$ and model rejection factors for model of astrophysical neutrino sources

\begin{tabular}{lclll}
\hline Model & \multicolumn{2}{l}{ Baikal } & \multirow{2}{*}{ AMANDA [23-25] } \\
\cline { 2 - 3 } & $v_{e}+v_{\mu}+v_{\tau}$ & $n_{90 \%} / N_{\mathrm{m}}$ & & $n_{90 \%} / N_{\mathrm{m}}$ \\
\hline $10^{-6} \times E^{-2}$ & 3.08 & 0.81 & 0.22 \\
SS Quasar [26] & 10.00 & 0.25 & 0.21 \\
SS05 Quasar [27] & 1.00 & 2.5 & 1.6 \\
SP u [28] & 40.18 & 0.062 & 0.054 \\
SP 1 [28] & 6.75 & 0.37 & 0.28 \\
P $p \gamma$ [29] & 2.19 & 1.14 & 1.99 \\
M pp $+p \gamma$ [30] & 0.86 & 2.86 & 1.19 \\
MPR [31] & 0.63 & 4.0 & 2.0 \\
SeSi [32] & 1.18 & 2.12 & - \\
\hline
\end{tabular}

obtained assuming an uncertainty in signal detection of $24 \%$ and a background of zero events.

A model of astrophysical neutrino sources, for which the total number of expected events, $N_{\mathrm{m}}$, is large than $n_{90 \%}$, is ruled out at $90 \% \mathrm{CL}$. Table 1 represents event rates and model rejection factors (MRF) $n_{90} / N_{\mathrm{m}}$ for models of astrophysical neutrino sources obtained from our search, as well as MRF obtained recently by the AMANDA collaboration [23-25].

For an $E^{-2}$ behavior of the neutrino spectrum and a flavor ratio $v_{e}: v_{\mu}: v_{\tau}=1: 1: 1$, the $90 \% \mathrm{CL}$ upper limit on the neutrino flux of all flavors obtained with the Baikal neutrino telescope NT200 (1038 days) is

$E^{2} \Phi<8.1 \times 10^{-7} \mathrm{~cm}^{-2} \mathrm{~s}^{-1} \mathrm{sr}^{-1} \mathrm{GeV}$.

For the resonant process with the resonant neutrino energy $E_{0}=6.3 \times 10^{6} \mathrm{GeV}$ the model-independent limit on $\overline{v_{e}}$ is

$\Phi_{\overline{v_{e}}}<3.3 \times 10^{-20} \mathrm{~cm}^{-2} \mathrm{~s}^{-1} \mathrm{sr}^{-1} \mathrm{GeV}^{-1}$.

Fig. 7 (left panel) shows our upper limit on the all flavor $E^{-2}$ diffuse flux (2) as well as the model-independent limit on the resonant $\bar{v}_{e}$ flux (diamond) (3). Also shown are the limits obtained by AMANDA [23-25] and MACRO [33], theoretical bounds obtained by Berezinsky (model independent (B) [34] and for an $E^{-2}$ shape of the neutrino spectrum $\left(\mathrm{B}\left(E^{-2}\right)\right)$ [35], by Waxman and Bahcall (WB) [36], by Mannheim et al. (MPR) [31], predictions for neutrino fluxes from topological defects (TD) [32], prediction on diffuse flux from AGNs according to Nellen et al. (NMB) [37], as well as the atmospheric conventional neutrino fluxes [38] from horizontal and vertical directions $((v)$ upper and lower curves, respectively) and atmospheric prompt neutrino fluxes $\left(v_{\mathrm{pr}}\right)$ obtained by Volkova et al. [39]. The right panel of Fig. 7 shows our upper limits (solid curves) on diffuse fluxes from AGNs shaped according to the model of Stecker and Salamon (SS, SS05) [26,27] and of Semikoz and Sigl (SeSi) [32], according to Table 1. 

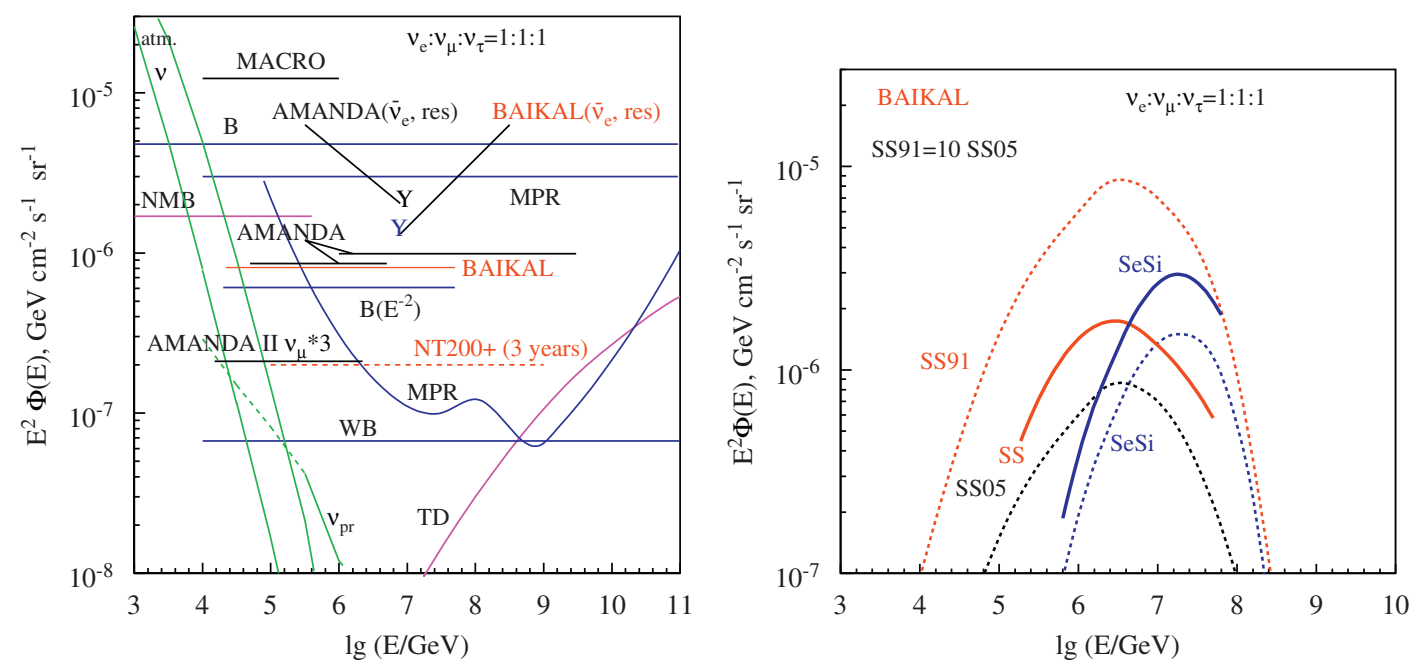

Fig. 7. Left panel: all-flavor neutrino flux predictions in different models of neutrino sources compared to experimental upper limits to $E^{-2}$ fluxes obtained by this analysis and other experiments (see text). Also shown is the sensitivity expected for three live years of the new telescope NT200+ [5,42]. Right panel: Baikal experimental limits compared to two model predictions. Dotted curves: predictions from model SS [26], SeSi [32] and SS05 [27]. Full curves: obtained experimental upper limits to spectra of the same shape. Model SS is excluded (MRF $=0.25)$, model SeSi is not $(\mathrm{MRF}=2.12$ ).

\section{A prototype device for acoustic neutrino detection in Lake Baikal}

Since several years, feasibility studies towards acoustic detection of high-energy cascades are performed in Lake Baikal. It was shown [40] that at stationary and homogeneous meteorological conditions the integral noise power in the frequency range $20-50 \mathrm{kHz}$ can reach levels as low as $1 \mathrm{mPa}$.

In April 2006, a digital hydro-acoustic device with four input channels was put in permanent operation as a part of NT200+ [41]. Analysis of data taken with this device during April-May 2006 shows that most acoustic pulses are located in the vicinity of the horizon plane. From the region $\pm 45^{\circ}$ around the opposite zenith, no event with bipolar pulse form, which is expected for acoustic signals from cascades, has been observed. The results of the measurements have demonstrated the feasibility of the proposed acoustic pulse detection technique in searching for signals from ultrahigh-energy cascades.

\section{Towards a $\mathbf{k m}^{3}$ detector in Lake Baikal}

The construction of NT200+ is a first step towards a $\mathrm{km}^{3}$-scale Baikal neutrino telescope. Such a detector could be made of building blocks similar to NT200+, but with NT200 replaced by a single string, still allowing separation of high-energy neutrino induced cascades from background. It will contain a total of $1300-1700 \mathrm{OMs}$, arranged at $90-100$ strings with $12-16 \mathrm{OMs}$ each, and a length of $300-350 \mathrm{~m}$. Interstring distance will be $\approx 100 \mathrm{~m}$. The effective volume for detection of cascades with energy above $100 \mathrm{TeV}$ is $0.5-0.8 \mathrm{~km}^{3}$.

The existing NT200+ allows to verify all key elements and design principlest of the $\mathrm{km}^{3}$ (Gigaton-Volume) Baikal telescope. Next milestone of the ongoing $\mathrm{km}^{3}$-telescope research and development work (R\&D) will be spring 2008: installation of a "new technology" prototype string as a part of NT200+. This string will consist of 12 optical modules and a FADC based measuring system. Three issues, discussed in the remainder of this paper, have been investigated in 2007, and will permit installation of this prototype string: (1) increase of underwater (uw) data transmission bandwidth, (2) in situ study of FADC PMpulses, (3) preliminary selection of optimal PM.

\subsection{Modernization of data acquisition system}

The basic goal of the NT200+ DAQ modernization is a substantial increase of uw-data rate to allow for transmission of significant FADC data rate, and also for a more complex trigger concept (e.g. lower thresholds and topological trigger). In a first step, in 2005 a high speed data/control tcp/ip connection between the shore station and the central uw-PCs (data center) had been established (full multiplexing over a single pair of wires, with a hot spare) $[4,5,42]$, based on DSL modems (FlexDSL). In 2007, the communication on the remaining segment uw-PC string controller was upgraded using the same approach. The basic elements are new string-controllers (handling TDC/ ADC-readout) with an ethernet-interface, connected by a DSL modem to the central uw-DSL unit (3 DSL modems, max. 2 Mbps each), connected by ethernet to the uw-PCs. The significant increase in uw-data rate (string to uw-PC) provided the possibility to operate the new prototype FADC system.

\subsection{Prototype on a FADC based system}

A prototype FADC readout system was installed during the Baikal expedition 2007. It should yield input for the design of the $2008 \mathrm{~km}^{3}$ prototype string (FADC), such as 
optimal sampling time window, dynamic range, achievable pulse parameter precisions, algorithms for online data handling, estimation of true bandwidth needs. These data will also be useful to decide about the basic DAQ/ Triggering approach for the $\mathrm{km}^{3}$-detector: at this stage, both a complex FADC based, as well as a classical TDC/ADC approach seem feasible.

The FADC prototype is located at the top of the second outer string, see Fig. 8. It includes two optical modules with up-looking PM R8055, a slow control module and a FADC sphere. The FADC sphere consists of two $250 \mathrm{MHz}$ FADCs, with USB connection to an embedded PC104 computer emETX-i701, and a counter board MPC148. The standard string trigger (twofold channel coincidence) is used as FADC trigger. Data are transferred via local ethernet and the DSL-link of the second string. Data analysis from FADC prototype is in progress. Fig. 9 shows examples of FADC pulses for different classes of events. The top panel gives a 1-photoelectron (p.e.) noise hit, for scale. A muon trigger (multi-p.e.) is given in the middle panel; the bottom panel shows an interesting event due to backward illumination by an intense calibration laser, located $\sim 140 \mathrm{~m}$ away. The PM orientation opposite to the calibration laser explains the significant signal duration
(>100 ns), illustrating the light scattering influence on particle detection for large distances.

\subsection{PM selection for the $\mathrm{km}^{3}$ prototype string}

Selection of the optimal PM type for the $\mathrm{km}^{3}$ telescope is a key question of detector design. Assuming similar values for time resolution and linearity range, the basic criteria of PM selection is its effective sensitivity to Cherenkov light, determined as the fraction of registered photons per photon flux unit. It depends on photocathode area, quantum efficiency, and photoelectron collection efficiency. We compared effective sensitivities of Hamamatsu R8055 (13 in. photocathode diameter) and XP1807 (12 in.) with QUASAR-370 (14.6 in.) [43], which was successfully operated in NT200 over more than 15 years. In laboratory we used blue LEDs $(470 \mathrm{~nm})$, located at $150 \mathrm{~cm}$ distance from the PM. Underwater measurements are done for 2 R8055 and 2 XP1807, installed permanently as two NT200channels, which are illuminated by the external laser calibration source [42], located 160-180 m away. Preliminary results of these effective PM sensitivity measurements are given in Fig. 10, and show relatively small deviations. Smaller size (R8055, XP1807) tends to be compensated by

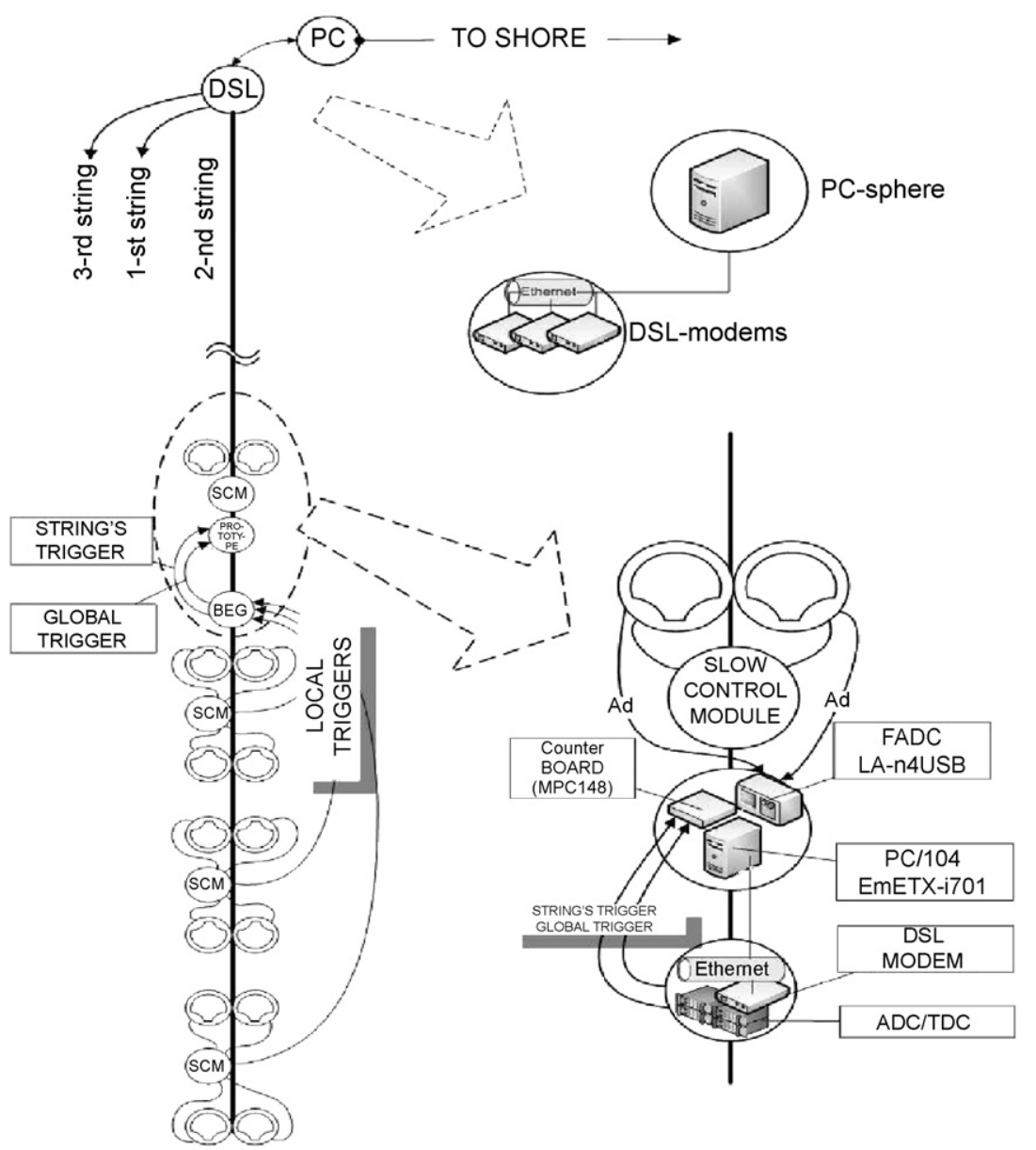

Fig. 8. The external string readout/control system, with the FADC prototype for two $13 \mathrm{in}$. PMs. 

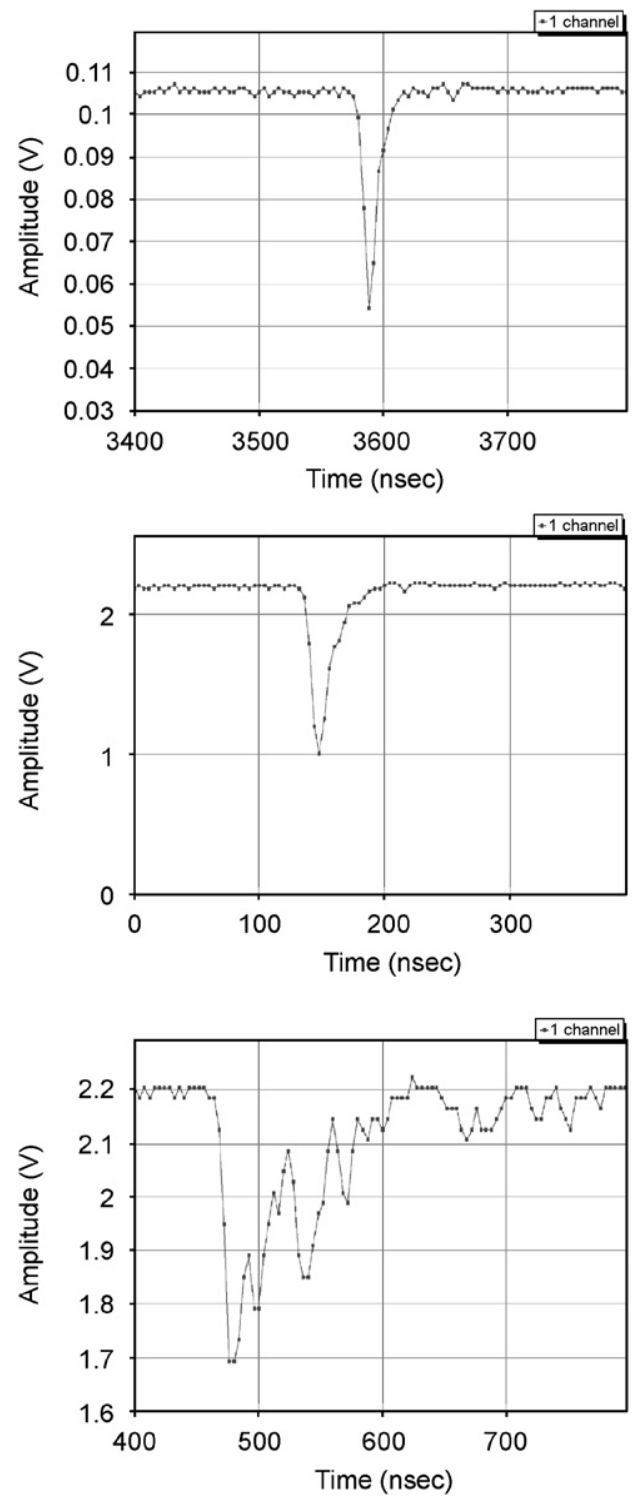

Fig. 9. Examples of 13 in. PM pulses from the FADC prototype, see Fig. 8. Top: noise pulse, middle: muon event, bottom: laser pulse (backward illumination of PM).

larger photocathode sensitivities. In addition, we emphasize the advantage of a spherical shape (as QUASAR-370); we are investigating the angular integrated sensitivity looses due to various deviations from that optimum.

\section{Conclusion}

The Baikal neutrino telescope NT200 is taking data since April 1998. The upper limit obtained for a diffuse $\left(v_{e}+v_{\mu}+v_{\tau}\right)$ flux with $E^{-2}$ shape is $E^{2} \Phi=8.1 \times$ $10^{-7} \mathrm{~cm}^{-2} \mathrm{~s}^{-1} \mathrm{sr}^{-1} \mathrm{GeV}$. The limits on fast magnetic monopoles and on additional muon flux induced by WIMPs annihilation at the center of the Earth belong to the most stringent limits existing to date. The limit on a $\overline{v_{e}}$ flux at the resonant energy $6.3 \times 10^{6} \mathrm{GeV}$ is presently the most stringent.

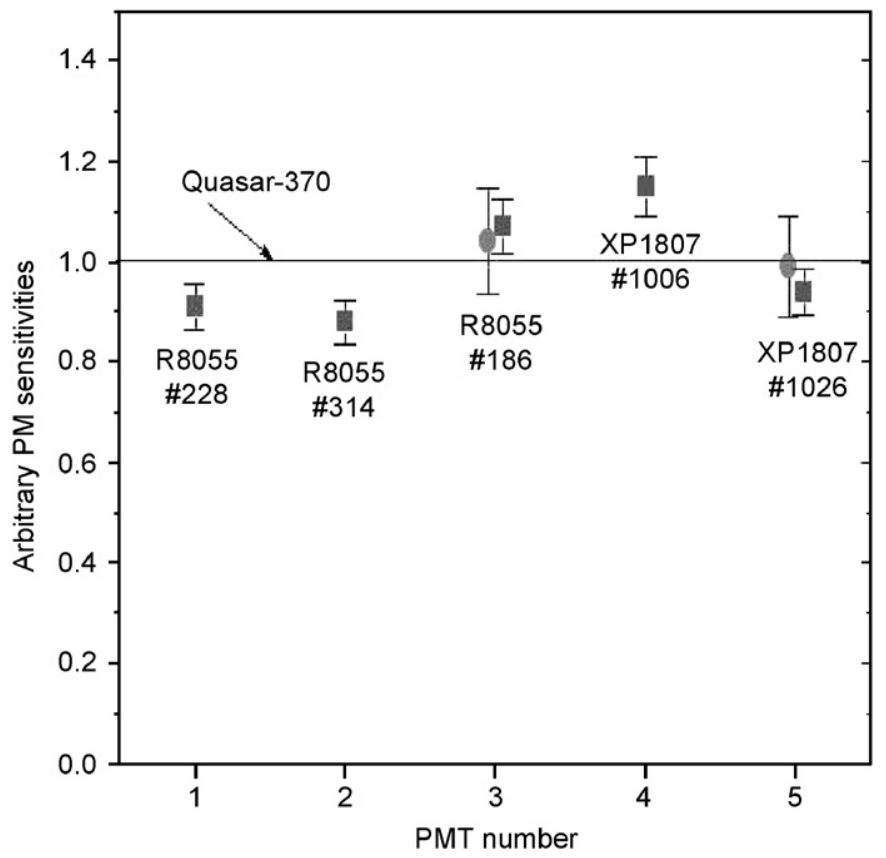

Fig. 10. Ratio of effective sensitivity of large area PMs R8055/13 in. and XP1807/12 in. to QUASAR-370/14.6in. Laboratory measurements (squares), in situ (dots).

To extend the search for diffuse extraterrestrial neutrinos with higher sensitivity, NT200 was significantly upgraded to NT200+, a detector with about 5 Mton enclosed volume, which takes data since April 2005 [5,42]. The three-year sensitivity of NT200+ to the all-flavor neutrino flux is approximately $2 \times 10^{-7} \mathrm{~cm}^{-2} \mathrm{~s}^{-1} \mathrm{sr}^{-1} \mathrm{GeV}$ for $E>10^{2} \mathrm{TeV}$ (shown in Fig. 7).

For a $\mathrm{km}^{3}$-scale detector in Lake Baikal, R\&D activities are in progress. The NT200+ detector is, beyond its better physics sensitivity, used as an ideal testbed for critical new components. Modernization of the NT200+ DAQ allowed to install a prototype FADC PM readout. Six large area hemispherical PMs have been integrated into NT200+ (2 Photonis XP1807/12 in. and 4 Hamamatsu R8055/ 13 in.), to facilitate an optimal PM choice. A prototype new technology string will be installed in spring 2008 and a $\mathrm{km}^{3}$-detector Technical Design Report is planned for fall 2008.

\section{Acknowledgments}

This work was supported by the Russian Ministry of Education and Science, the German Ministry of Education and Research and the Russian Fund of Basic Research (Grants 05-02-17476, 05-02-16593, 07-02-10013 and 07-0200791), and by the Grant of President of Russia NSh4580.2006.2. and by NATO-Grant NIG-9811707 (2005).

\section{References}

[1] I. Belolaptikov, et al., Astropart. Phys. 7 (1997) 263.

[2] V. Aynutdinov, et al., Nucl. Phys. (Proc. Suppl.) B 143 (2005) 335. 
[3] V. Aynutdinov, et al., Astropart. Phys. 25 (2006) 140.

[4] V. Aynutdinov, et al., in: Proceedings of V International Conference on Non-Accelerator New Physics, Dubna, Russia, June 7-10, 2005.

[5] V. Aynutdinov, et al., Nucl. Instr. and Meth. A 567 (2006) 433.

[6] V. Aynutdinov, et al., in: Proceedings of the 30th ICRC (icrc1084), Merida, 2007, arXiv.org: astro-ph/0710.3063.

[7] V. Aynutdinov, et al., in: Proceedings of the 30th ICRC (icrc1088), Merida, 2007, arXiv.org: astro-ph/0710.3064.

[8] V. Balkanov, et al., Astropart. Phys. 12 (1999) 75.

[9] V. Aynutdinov, et al., Int. J. Mod. Phys. B 20 (2005) 6932.

[10] V. Balkanov, et al., Nucl. Phys. (Proc. Suppl.) B 91 (2001) 438.

[11] V. Agrawal, T. Gaisser, P. Lipari, T. Stanev, Phys. Rev. D 53 (1996) 1314.

[12] M. Boliev, et al., Nucl. Phys. (Proc. Suppl.) 48 (1996) 83; O. Suvorova, arXiv.org: hep-ph/9911415, 1999.

[13] M. Ambrosio, et al., Phys. Rev. D 60 (1999) 082002.

[14] S. Desai, et al., Phys. Rev. D 70 (2004) 083523; S. Desai, et al., Phys. Rev. D 70 (2004) 109901.

[15] J. Ahrens, et al., 2005, arXiv.org: astro-ph/0509330.

[16] K. Antipin, et al., in: Proceedings of the First Workshop on Exotic Physics with Neutrino Telescopes, Uppsala, Sweden, September 20-22, 2006, p. 34.

[17] K. Antipin, et al., in: Proceedings of the First Workshop on Exotic Physics with Neutrino Telescopes, Uppsala, Sweden, September 20-22, 2006, p. 80.

[18] I. Belolaptikov, et al., Baikal Collaboration, in: 26th ICRC, vol. 2, Salt Lake City, 1999, p. 340.

[19] S. Orito, et al., Phys. Rev. Lett. 66 (1991) 1951.

[20] M. Ambrosio, et al., MACRO Collaboration, arXiv.org: hep-ex/ 02007020, 2002.

[21] P. Niessen, C. Spiering, AMANDA Collaboration, in: 27th ICRC, vol. 4, Hamburg, 2001, p. 1496.
[22] H. Wissing, et al., Ice Cube Collaboration, in: Proceedings of the 30th ICRC, Merida, 2007.

[23] M. Ackermann, et al., Astropart. Phys. 22 (2005) 127; M. Ackermann, et al., Astropart. Phys. 22 (2005) 339.

[24] M. Ackermann, et al., Astropart. Phys. 22 (2005) 339.

[25] M. Ackermann, et al., Phys. Rev. D 76 (2007) 042008.

[26] F. Stecker, M. Salamon, Space Sci. Rev. 75 (1996) 341.

[27] F. Stecker, Phys. Rev. D 72 (2005) 107301.

[28] A. Szabo, R. Protheroe, in: V.J. Stenger, et al. (Eds.), Proceedings of the High Energy Neutrino Astrophysics, Honolulu, Hawaii, 1992.

[29] R. Protheroe, arXiv.org: astro-ph/9612213.

[30] K. Mannheim, Astropart. Phys. 3 (1995) 295.

[31] K. Mannheim, R. Protheroe, J. Rachen, Phys. Rev. D 63 (2001) 023003.

[32] D. Semikoz, G. Sigl, arXiv.org: hep-ph/0309328.

[33] M. Ambrosio, et al., Nucl. Phys. (Proc. Suppl.) B 110 (2002) 519.

[34] V. Berezinsky, et al., in: Astrophysics of Cosmic Rays, Elsevier Science, North-Holland, Amsterdam, 1990.

[35] V. Berezinsky, arXiv.org: astro-ph/0505220, 2005.

[36] E. Waxman, J. Bahcall, Phys. Rev. D 59 (1999) 023002.

[37] L. Nellen, K. Mannheim, P. Biermann, Phys. Rev. D 47 (1993) 5270.

[38] L. Volkova, Yad. Fiz. 31 (1980) 1510.

[39] L. Volkova, G. Zatsepin, Phys. Lett. B 462 (1999) 211.

[40] V. Aynutdinov, et al., Int. J. Mod. Phys. A 21S1 (2006) 117; V. Aynutdinov, et al., Acoust. Phys. 52 (5) (2006) 495.

[41] V. Aynutdinov, et al., in: Proceedings of the 30th ICRC (icrc0639), Merida, 2007, arXiv.org: astro-ph/0710.3113.

[42] V. Aynutdinov, et al., in: Proceedings of the 29th International Cosmic Ray Conference, Pune, India, August 3-10, 2005, arXiv.org: astro-ph/0507715.

[43] R. Bagduev, et al., Nucl. Instr. and Meth A 420 (1999) 138. 